\title{
Tenencia de perros de compañía. Beneficios para la salud psico-emocional de los pacientes con Diabetes Mellitus Tipo 2 de la mediana edad
}

\section{Dog's ownership. Benefits for the psycho-emotional health of middle-aged patients with Type 2 Diabetes Mellitus}

\author{
Beatriz Hugues Hernandorena ${ }^{1,2}$, Aimée M. Álvarez Álvarez ${ }^{1}$, \\ Lizet Castelo Elias-Calles ${ }^{1}$, Loraine Ledón Llanes ${ }^{1}$, \\ Madelin Mendoza Trujillo ${ }^{1}$, Emma Domínguez Alonso ${ }^{1}$
}

\section{Resumen}

La tenencia de perros de compañía ejerce un efecto positivo en la prevención y recuperación de la salud mental en las personas. Los pacientes con diabetes mellitus tipo 2 (DM2) de la mediana edad suelen padecer estas alteraciones por lo que la tenencia de estos animales pudiera ser de utilidad. Con el objetivo de describir algunas variables de la salud psicoemocional (ansiedad, depresión vulnerabilidad al estrés) en personas con DM2 y los efectos de la tenencia sobre aspectos de la satisfacción personal y las motivaciones para la realización de actividades físicas, se realizó un estudio descriptivo que incluyó 24 personas con perros y 24 sin ellos, quienes se atendían en el Centro de Atención al Diabético de La Habana. No se encontró diferencias significativas entre pacientes de mediana edad con DM2 con o sin perros de compañía a partir de indicadores de la estabilidad psíquica, pero se observó una tendencia de menores niveles de ansiedad en aquellos con perros de compañía. Los pacientes con perros de compañía mostraron gran satisfacción personal y percibieron beneficios en la salud psicoemocional con respecto a las motivaciones para la realización de actividades físicas a partir de la tenencia de su animal.

Palabras clave: tenencia de animales de compañía; salud física; actividades físicas

\footnotetext{
${ }^{1}$ Instituto Nacional de Endocrinología (INEN), La Habana, Cuba

${ }^{2}$ E-mail: bettymig@infomed.sld.cu
} 
The possession of companion dogs has a positive effect on the prevention and recovery of mental health in people. Patients with diabetes mellitus type 2 (DM2) of middle age often suffer from these alterations so that the possession of these animals could be useful. In order to describe some variables of psycho-emotional health (anxiety, depression, vulnerability to stress) in people with DM2 and the effects of tenure of dogs on aspects of personal satisfaction and the motivations for carrying out physical activities, a descriptive study was carried out. The work included 24 people with dogs and 24 without them, who were patients of the Diabetic Care Centre of Havana. No significant differences were found between middle-aged patients with DM2 with or without companion dogs in relation to indicators of psychic stability, but a trend of lower levels of anxiety was observed in those with companion dogs. Patients with companion dogs showed great personal satisfaction and perceived benefits in psycho-emotional health with respect to the motivations for carrying out physical activities due to the possession of the animal.

Key words: pet ownership; physical health; physical activities

\section{INTRODUCCIÓN}

La tenencia de perros de compañía brinda un gran beneficio en la vida de las personas (Díaz et al., 2015). La convivencia con ellos ejerce un efecto socializador (Gómez et al., 2017; Herzog, 2011) y otros beneficios relacionados con el estado psicoemocional (estrés, ansiedad, depresión) (González y Landero, 2011). De hecho, ha sido demostrado que los que coexisten con ellos asisten menos a las visitas médicas en comparación con aquellos que no los tienen (O'Haire, 2010).

La diabetes mellitus tipo 2 (DM2) es una enfermedad crónica no transmisible, que conlleva un seguimiento a largo plazo (Domínguez et al., 2010). Durante el transcurso de la enfermedad se pueden generar vulnerabilidades psicosociales, sobre todo en los comprendidos en la mediana edad (Hernández et al., 2011). Esta enfermedad tiene repercusión en la interacción familiar. Se reconoce que los cambios vividos desde el punto de vista psíquico y emocional, así como su expresión en las relaciones interpersonales (pareja, familia, compañeros de trabajo), de las personas con DM2 constituyen el contenido fundamental de las preocupaciones y del sentido de insatisfacción en estas personas (García et al., 2009, 2015; Ledón, 2012). La depresión, la ansiedad y el estrés son factores de riesgo para la DM2 y viceversa (Ledón, 2008).

La tenencia de perros de compañía podría ser parte de la atención integral de personas con DM2. Se considera que ayudan a disminuir las alteraciones psicológicas, reducen el sentimiento de soledad e incrementan el de intimidad, la autoestima y mejoran la aptitud física y la extroversión (Portuondo, 2012, 2016); asimismo, promueve la relación y socialización entre las personas desconocidas, que se vuelven más solidarias y comunicativas y facilitan la comunicación entre distintas generaciones dentro o fuera del marco familiar por ser un foco de interés común (Díaz et al., 2015a,b). Sin embargo, también existen efectos negativos que están relacionados con la transmisión de enfermedades zoonóticas, mordeduras y arañazos y los traumas psicológicos que sufren las personas ante su muerte. 
Teniendo en cuenta lo expresado, se llevó a cabo el presente estudio para describir algunas variables psicoemocionales (ansiedad, depresión, vulnerabilidad al estrés) en pacientes con DM2 con y sin perros de compañía atendidas en el Centro de Atención al Diabético de La Habana, Cuba, así como los efectos de la tenencia de perros de compañía sobre aspectos de la salud psicoemocional y su relación con la realización de actividades físicas.

\section{Materiales y Métodos}

Se llevó a cabo un estudio descriptivo, transversal, utilizando metodología cuantitativa de investigación, en las áreas de consultas del Centro de Atención al Diabético de La Habana, Cuba, entre mayo de 2013 y diciembre de 2016.

La muestra quedó integrada por 48 pacientes de la mediana edad (40-59 años) con diagnóstico de DM2. Se definió un grupo de estudio compuesto por 24 pacientes, hombres y mujeres, que tenían a su cargo perros de compañía (Tenencia PC) por lo menos desde hace un año. Así mismo, un grupo de comparación de 24 pacientes, hombres y mujeres, que no tuvieron a su cargo perros de compañía (No tenencia PC).

Los pacientes que pertenecieron a cada grupo fueron pareados según su género y edad (rango de \pm 3 años), tiempo de diagnóstico de la DM2 (rango de \pm 3 años) y tipo de tratamiento de la DM2 (insulina, hipoglucemiantes orales, ambos o dieta), para lograr que la muestra fuera homogénea. Este estudio es parte de un estudio global sobre tenencia de perros en pacientes de mediana edad con DM2, cuya primera parte ha sido publicada (Hugues et al., 2018).

Las variables de la salud psicoemocional que se exploraron fueron la ansiedad, la depresión y la vulnerabilidad al estrés. Para la evaluación de estas variables se utilizaron los siguientes instrumentos (González, 2007) de forma autoadministrada (respondido por el propio paciente):

- Modelo de autoanálisis de Cattell: cuestionario dirigido a evaluar nivel de ansiedad y explorar los factores que contribuyen a la misma.

- Auto-escala de depresión de Zung y Conde: cuestionario que explora la frecuencia de aparición de un grupo de manifestaciones que dan cuenta de la presencia de depresión.

- Test de vulnerabilidad al estrés de Miller y Smith: cuestionario dirigido a explorar los estilos de conductas que conducen a comportamientos saludables o que favorecen la aparición de enfermedades como consecuencia de lo vulnerable que se hace el individuo al efecto estresor de cualquier agente, cuando presenta estilos de comportamiento proclives a enfermar.

Para describir los efectos de la tenencia de perros de compañía sobre aspectos de la salud psicoemocional y la realización de actividades físicas, se aplicó un instrumento confeccionado por el propio colectivo de investigadores (cuestionario sobre tenencia de animales de compañía y diabetes mellitus tipo 2 ) en el que se exploraban estos y otros aspectos.

Se obtuvieron distribuciones de frecuencia y porcentajes de las variables cualitativas, y la mediana y porcentajes para las variables cuantitativas. La asociación entre estas variables y la tenencia de animales de compañía se exploró mediante la prueba Chi cuadrado. En todos los casos se utilizó un nivel de significancia de 0.05 . Los datos se procesaron estadísticamente mediante el empleo del sistema SPSS v. 19.0.

La participación de los sujetos en el estudio fue voluntaria y anónima. A cada persona que cumplió con los criterios de inclusión muestral se le solicitó su participación en el estudio, previa información (oral) de sus objetivos, relevancia, características de la 
Cuadro 1. Distribución porcentual de las variables psicoemocionales de los pacientes con DM2 ( $\mathrm{n}=24$ por grupo)

\begin{tabular}{lrcccccc}
\hline \multirow{2}{*}{ Grupos } & & \multicolumn{2}{c}{ Tenencia PC } & \multicolumn{2}{c}{ No tenencia PC } & \multicolumn{2}{c}{ Total } \\
\cline { 2 - 8 } & $\mathrm{n}$ & $\%$ & $\mathrm{n}$ & $\%$ & $\mathrm{n}$ & $\%$ \\
\hline Ansiedad & $\begin{array}{r}\text { Muy elevada a } \\
\text { moderada }\end{array}$ & 5 & 20.8 & 13 & 54.2 & 18 & 37.5 \\
Leve & 4 & 16.7 & 1 & 4.2 & 5 & 10.4 \\
& $\begin{array}{r}\text { Esencialmente } \\
\text { normal }\end{array}$ & 13 & 54.2 & 9 & 37.5 & 22 & 45.8 \\
& Sub-motivado & 2 & 8.3 & 1 & 4.2 & 3 & 6.3 \\
Depresión & No depresivo & 7 & 29.2 & 7 & 29.2 & 14 & 29.2 \\
& Leve & 13 & 54.2 & 10 & 41.7 & 23 & 47.9 \\
& Moderada & 3 & 12.5 & 4 & 16.7 & 7 & 14.6 \\
Severa & 1 & 4.2 & 3 & 12.5 & 4 & 8.3 \\
Vulnerabilidad & No vulnerable & 14 & 58.3 & 13 & 54.3 & 27 & 56.2 \\
al estrés & Vulnerable & 10 & 41.7 & 11 & 45.7 & 21 & 43.8 \\
\hline
\end{tabular}

Diferencias entre grupos sin significancia estadística

participación y beneficios. A todos los pacientes se les informó que la decisión de no participar en el estudio no tenía efecto negativo alguno en la atención que recibiría. Toda la información recogida se manejó de forma confidencial, sin registro de identidad personal. La información fue codificada.

\section{Resultados y Discusión}

La distribución porcentual de los pacientes con DM2 de acuerdo con las variables psicosociales se describen en el Cuadro 1. No se encontró diferencia significativa entre grupos para las variables en estudio. No obstante, el $54.2 \%$ de personas sin tenencia de perros de compañía presentó grados de ansiedad muy elevado a moderado, mientras que se encontró esta misma frecuencia en pacientes que sí los tenían cuyo nivel de ansiedad era esencialmente normal. En forma similar, el grado de depresión tendió a ser más severo en pacientes sin perros de compañía.
Cuadro 2. Evaluación de la satisfacción personal en relación con la tenencia de perros de compañía

\begin{tabular}{lcc}
\hline Satisfacción personal & $\mathrm{n}$ & $\%$ \\
\hline $\begin{array}{l}\text { - Cuido mejor de mi } \\
\text { salud }\end{array}$ & 24 & 100.0 \\
$\begin{array}{l}\text { - Provoca bienestar } \\
\text { cuidarlo }\end{array}$ & 20 & 83.3 \\
$\begin{array}{l}\text { - Disminuye } \\
\text { preocupaciones DM2 }\end{array}$ & 20 & 83.3 \\
$\begin{array}{l}\text { - Libera de las tensiones, } \\
\text { el estrés y la ansiedad }\end{array}$ & 19 & 79.1 \\
\hline
\end{tabular}

Los resultados de la influencia del perro de compañía sobre aspectos de la salud psicoemocional y su relación con la realización de actividades físicas se muestran en los cuadros 2, 3 y 4 . Con relación a la satisfacción personal, es de destacar que las cua- 
Cuadro 3. Evaluación de la salud psicoemocional con relación a la tenencia de perros de compañía $(n=24)$

\begin{tabular}{lcc}
\hline Satisfacción personal & $\mathrm{n}$ & $\%$ \\
\hline Beneficios & & \\
$\quad$ Muy beneficioso & 19 & 79.2 \\
$\quad$ Beneficioso & 5 & 20.8 \\
Mejora estado psico- & & \\
emocional & & \\
Sí & 17 & 70.8 \\
No & 3 & 12.5 \\
No sabe & 4 & 16.7 \\
\hline
\end{tabular}

Cuadro 4. Evaluación de la actividad física en relación con la tenencia de perros de compañía

\begin{tabular}{lcc}
\hline Caminatas $^{1}$ & $\mathrm{n}$ & $\%$ \\
\hline - Me mantienen & 13 & 100.0 \\
$\quad$ saludable & & \\
- Las hago por el animal & 11 & 84.6 \\
- Disfruto de su & 10 & 76.9 \\
$\quad$ compañía & & \\
- Me relajan & 9 & 69.2 \\
\hline
\end{tabular}

${ }^{1}$ Sobre 13 respuestas

tro respuestas fueron muy consistentes y denotan los beneficios que les brinda la convivencia con estos animales (Cuadro 2). La respuesta unánime sobre 'cuido mejor de mi salud' indica lo que representa la tenencia de estos animales a sus dueños con respecto a su salud psicoemocional y física.

Sobre los efectos de la tenencia del perro y la salud psicoemocional (Cuadro 3), los pacientes respondieron que les resultaba muy beneficioso (79.2\%) o beneficioso (20.8\%), y ninguno mostró insatisfacción. Así mismo, la mayoría (70.8\%) admitió que sus animales contribuían a mejorar su estado psico-emocional. Resultados similares fueron hallados por este equipo (Hugues et al., 2016), en el que incluyeron 85 personas con DM2 de la mediana edad, de las cuales el $84 \%$ expresó que este vínculo les resultaba muy beneficioso y el $86 \%$ afirmó que mejoraba su estado psicoemocional.

Mocoso (2009) menciona que la relación entre los animales de compañía y sus dueños es «un mutuo beneficio y una relación dinámica entre las personas y otros animales que es influenciada por comportamientos que son esenciales para la salud y el bienestar de ambos», lo cual concuerda con los resultados del presente estudio. Así mismo, Hosey y Melfi (2014) realizaron una amplia revisión bibliográfica sobre el efecto beneficioso que produce la tenencia de animales de compañía sobre aspectos de la salud psicoemocional de sus responsables.

El efecto de la actividad física (caminatas con el perro fuera de la casa) sobre la salud psicoemocional se muestra en el Cuadro 4. Hernández et al. (2015) resaltan la importancia del ejercicio físico como parte del tratamiento integral de la DM; sin embargo no siempre los pacientes se sienten en condiciones de realizarla o no encuentran motivación para ello. La literatura científica plantea que la tenencia de los perros resulta un estímulo, no solo para realizar actividades físicas como las caminatas de forma sistemática, sino para participar en competencias, efectuar paseos y deportes en familia (Cirulli, 2011; Tabares, 2013; Zaldívar, 2014). Los resultados del presente estudio muestran el efecto relajante y socializador que ejercieron los perros para sus dueños, además de ser «el motor impulsor» y el aliciente para la ejercitación que requieren estas personas. 


\section{Conclusiones}

- No se encontraron diferencias significativas entre pacientes de mediana edad con diabetes mellitus tipo 2 con o sin perros de compañía a partir de indicadores de mejor estabilidad psíquica (ansiedad, depresión, vulnerabilidad al estrés); no obstante, se observó una tendencia de menores niveles de ansiedad en aquellos con perros de compañía.

- Los pacientes con perros de compañía mostraron gran satisfacción personal y percibieron beneficios en la salud psicoemocional con respecto a las motivaciones para la realización de actividades físicas a partir de la tenencia de su animal.

\section{Literatura Citada}

1. Cirulli F, Borgi M, Berry A, Francia N, Alleva E. 2011. Animal-assisted interventions as innovative tools for mental health. Ann I Super Sanita 47: 341348. doi: 10.4415/Ann 11_04_04.

2. Díaz M, Olarte MA, Martin J. $2015 a$. Antrozoología: definiciones, áreas de desarrollo y aplicaciones prácticas para profesionales de la Salud. Eur Scientific J 2: 185-210.10.19044/esj.2015.v11n10p\%25p

3. Díaz M, Olarte MA, Martin J. $2015 b$. Perfiles básicos del humano compañero del perro: Una revisión teórica en antrozoología guiada por el enfoque multimodal. Rev Argentina Cienc Comportamiento 7: 79-89.

4. Domínguez E, Seuc A, Díaz O, Aldana D. 2010. Esperanza de vida saludable asociada a la diabetes en Cuba: años 1990 y 2003. Rev Cubana Endocrinol 21: 13-34.

5. García R, García M, Suárez R, Domínguez E. 2009. Problemática laboral en un grupo de personas con diabetes mellitus. Rev Cubana Endocrinol 20: 89-103.
6. García Y, Casanova D, Rodríguez BC. 2015. Particularidades de los pensamientos intrusos y su supresión en pacientes con diabetes mellitus tipo 2. Rev Cubana Endocrinol 26: 6-20.

7. Gómez LF, Aterhortúa CG, Orozco SC. 2007. La influencia de las mascotas en la vida humana. Rev Colomb Cienc Pec 20:377-386.

8. González, F. 2007. Instrumentos de evaluación psicológica. La Habana, Cuba: Ed Ciencias Médicas. 424 p.

9. González MT, Landero R. 2011. Diferencias en estrés percibido, salud mental y física de acuerdo al tipo de relación humano-perro. Rev Colomb Psicol 20: 75-86.

10. Hernández J, Licea ME, Castelo L. 2015. Algunas formas alternativas de ejercicio, una opción a considerar en el tratamiento de personas con diabetes mellitus. Rev Cubana Endocrinol 26: 77-92.

11. Hernández Y, Victoria CR, Jaimes J, Saavedra A, Tapanes A, Solares $V$. 2011. Calidad de vida en pacientes con diabetes mellitus tipo II. Rev Hosp Psiquiátrico Habana 8(3). [Internet]. Disponible en: http://www.revistahph.sld.cu/ hph3-2011/hph02311.html

12. Herzog H. 2011. The impact of pets on human health and psychological wellbeing. Fact, fiction, or hypothesis? Curr Dir Psychol Sci 20: 236-239. doi: 10.1177/ 0963721411415220

13. Hosey G, Melfi V. 2014. Human-animal interactions, relationships and bonds: a review and analysis of the literature. Int J Comp Sociol 27: 117-142.

14. Hugues B, Álvarez AM, Castelo L, Ledón L, Mendoza M, Domínguez E. 2016. Percepción de los beneficios de la tenencia de animales de compañía para las personas de la mediana edad con diabetes mellitus tipo 2. Rev Inv Vet Peru 27:225-232. doi: 10.15381/rivep.v27i2.11646

15. Hugues B, Álvarez A, Elias L, Ledón L, Mendoza M, Domínguez E. 2018. Tenencia de perros de compañía. Benefi- 
cios para la salud física de pacientes de la mediana edad con Diabetes Mellitus Tipo 2. Rev Inv Vet Perú 29: xxx-xxx.

16. Ledón L. 2008. El desafío de vivir con enfermedades endocrinas: algunas anotaciones para la atención en salud. Rev Cubana Endocrinol 19(2). [Internet]. Disponible en: http://scielo.sld.cu/pdf/ end/v19n2/end08208.pdf

17. Ledón L. 2012. Impacto psicosocial de la diabetes mellitus, experiencias, significados y respuestas a la enfermedad. Rev Cubana Endocrinol 23: 76-97.

18. Mocoso F. 2009. Caninoterapia para la atención de pacientes con evento cerebro vascular. Estudio piloto. Tesis de Maestría. Bogotá, Colombia: Univ. Sergio Arboleda. 94 p.
19. O'Haire M. 2010. Companion animals and human health: benefits, challenges, and the road ahead. J Vet Behav 5: 226234. doi: $10.1016 /$ j.jveb.2010.02.002

20. Portuondo Z. 2012. ¿Qué sabemos de perros? La Habana, Cuba: Ed. Gente Nueva. 59 p.

21. Portuondo Z. 2016. El perro naturaleza y cultura. La Habana, Cuba: Ed. Científico-Técnica.

22. Tabares C, Vicente F, Sánchez S, Gómez M. 2013. Estado del arte sobre los efectos de la terapia asistida con perros en el tratamiento de enfermos de Alzheimer. Int J Develop Educat Psychol 2: 271-282.

23. Zaldívar D. 2014. Recrearse de manera sana y productiva. Salud Vida 3(1). [Internet]. Disponible en: http:// www.saludvida.sld.cu/articulo/2014/08/27/ recrearse-de-manera-sana-y-productiva 